\title{
Dinâmica populacional do Metamasius hemipterus e Cosmopolites sordidus em cultivo de Musa sp., na cidade de Sinop-MT
}

\author{
Janaina De Nadai CORASSA ${ }^{1 *}$, Ivone Beatryz dos SANTOS $^{1}$, Tamires Silva DUARTE \\ ${ }^{1}$ Instituto de Ciências Agrárias e Ambientais, Universidade Federal de Mato Grosso, Sinop, MT, Brasil. \\ *E-mail: janadenadai@gmail.com
}

Recebido em fevereiro/2018; Aceito em setembro/2018.

\begin{abstract}
RESUMO: A espécie Metamasius hemipterus (Linnaeus, 1758) (Coleoptera: Curculionidae), recentemente vêm sendo associada a danos em pseudocaules de bananeira, assim como a coleobroca Cosmopolites sordidus (Germar, 1824) (Coleoptera: Curculionidae), praga-chave de bananeira, devido aos danos diretos e indiretos que resultam em queda de produtividade e rentabilidade ao produtor. O monitoramento da população destes insetos é de grande relevância no momento da tomada de decisão do manejo de pragas da cultura. Assim, objetivou-se com este estudo monitorar a dinâmica populacional de $C$. sordidus e $M$. hemipterus em plantio comercial de banana cv. Nanicão. Para monitoramento das espécies-praga, armadilhas do tipo pitfall modificadas iscadas com feromônio de agregação sintético e toletes de cana-de-açúcar foram distribuídas para captura de curculionídeos praga, no período de maio de 2012 a abril de 2013. Os dados avaliados mostraram que a frequência de captura de $C$. sordidus durante o período do experimento apresentou variação na densidade populacional do inseto nas duas épocas do ano, correspondentes as épocas de chuva e seca. A dinâmica populacional de C. sordidus, foi influenciada positivamente pela precipitação pluviométrica, apresentando maiores níveis populacional na época chuvosa, com picos nos meses de novembro de 2012, janeiro e fevereiro de 2013. Em relação a M. hemipterus observou-se que a população do inseto não sofreu qualquer influência de variáveis climáticas.
\end{abstract}

Palavras-chave: monitoramento, moleque-da-bananeira, broca-rajada.

\section{Population dynamics of Metamasius hemipterus and Cosmopolites sordidus, in cultivation of Musa sp., in the city of Sinop-MT}

\begin{abstract}
The specie Metamasius hemipterus (Linnaeus, 1758) (Coleoptera: Curculionidae) has recently been associated with damage to banana pseudoculars, as well as the Coleobroca Cosmopolites sordidus (Germar, 1824) (Coleoptera: Curculionidae), a key plague of banana because the direct and indirect damages that result in a fall in productivity and profitability to the producer. The monitoring of the population of these insects is of great relevance at the moment of the decision making of the pest management of the crop. The objective with this study was to monitor the populational dynamics of $C$. sordidus and $M$. hemipterus in commercial plantation of banana cv. Nanicão. Modified pitfall-baited pitfalls with synthetic aggregation pheromone and sugarcane rods were distributed to catch curcumin curculionídeos from May 2012 to April 2013. The data evaluated showed that the frequency of capture of $C$. sordidus during the experiment period showed variation in the population density of the insect in the two seasons of the year, corresponding to rainy and dry seasons. The population dynamics of C. sordidus were positively influenced by rainfall, presenting higher population levels in the rainy season, with peaks in November 2012, January and February of 2013. In relation to M. hemipterus, it was observed that the population of the insect was not influenced by climatic variables.
\end{abstract}

Keywords: monitoring, banana weevil, West Indian sugarcane borer.

\section{INTRODUÇÃO}

No Brasil, país ranqueado como quarto produtor mundial de banana (FAOSTAT, 2015), a cultura encontra-se disseminada em todos os estados, abrangendo desde cultivos de subsistência em áreas de agricultura familiar a grandes cultivos sistematizados, envolvendo moderna tecnologia.

Em 2016, o Estado de Mato Grosso cultivou área de 7.217 hectares de bananais, registrando produção total de 72.009 toneladas da fruta, contribuindo com cerca de $1 \%$ da produção nacional, que foi de 6.962.134 toneladas, em uma área plantada de 474.054 hectares (IBGE, 2017).

Entretanto, alguns problemas fitossanitários têm provocado prejuízos à cultura, proporcionando perdas de produtividade e de rentabilidade, travando o mercado da bananicultura nacional. Dentre esses problemas destacam-se as coleobrocas, como Cosmopolites sordidus (GERMAR, 1824) e Metamasius hemipterus (LINNAEUS, 1758) (COLEOPTERA: CURCULIONIDAE), comumente conhecidos como moleque-da-bananeira e broca-rajada, respectivamente.

Fancelli et al. (2015) destacam a espécie $C$. sordidus como a principal praga da cultura da banana. Suas larvas provocam severos danos no pseudocaule e rizoma das bananeiras ao construírem galerias, alimentando-se de seus tecidos e, interferindo negativamente no fluxo de seiva e absorção de nutrientes do solo, além de tornar as plantas mais susceptíveis a patógenos. Os adultos, de hábitos noturnos, são responsáveis principalmente pela reprodução, visto que não provocam 
danos diretos às plantas. As folhas de bananeiras tornam-se amareladas e com os bordos ondulados, além de perdas em tamanho e peso dos cachos de banana, em decorrência dos ataques. Gallo et al. (2002) destaca a ocorrência de C. sordidus em todos os Estados brasileiros e, que a presença de 12 larvas da espécie por bananeira pode provocar perdas entre 20 a $50 \%$ da produção total.

A espécies $M$. hemipterus, é considerada uma pragasecundária de bananeiras, contudo pode ser frequentemente encontrada em pseudocaules tombados, restos-culturais em decomposição e plantas com desenvolvimento limitado (BORGES et al., 2015). Kochemborger et al. (2016) relataram o primeiro registro de M. hemipterus em bananais no Estado do Acre, no qual foram observadas larvas alimentando-se de tecidos vivos das bananeiras e abrindo galerias nos pseudocaules. Na cidade de Sinop, Mato Grosso, Molin; Barreto (2012) relataram a ocorrência de $M$. hemipterus como inseto praga na cultura de Cocos nucifera. A espécie provoca danos também em cultivos de Dendê (Elaeis guineenses) (DIONISIO et al., 2015). O gênero Metamasius é relacionado por Zorzenon et al. (2000) como praga das culturas de canade-açúcar, bromeliáceas, musáceas, palmáceas, entre outras e, sua distribuição abrange todo o continente americano, desde os Estados Unidos até o Brasil.

O monitoramento periódico realizado por armadilhas atrativas é um procedimento necessário para a tomada de medidas de controle de pragas, pois proporciona $o$ conhecimento da dinâmica populacional e do nível de infestação de $C$. sordidus e $M$. hemipterus no bananal, tornando-se, portanto, uma alternativa para o manejo racional da cultura, com redução de custos, impacto ambiental e aumento da segurança do trabalhador rural.

Ante a insuficiência de informações relacionadas à dinâmica populacional do $C$. sordidus e M. hemipterus no bioma amazônico, objetivou-se com este trabalho realizar o monitoramento destes curculionídeos, em plantios comerciais de banana, no município de Sinop - MT, acompanhando sua ocorrência e densidade populacional em diferentes condições climáticas, correspondentes às estações seca e chuvosa, características da região, com vistas à produção de subsídios técnicos que contribuam no delineamento do manejo integrado e facilite as ações interventivas.

\section{MATERIAL E MÉTODOS}

O experimento foi instalado em um bananal comercial,

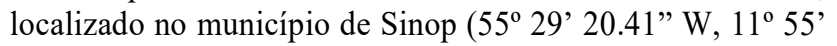
57,86 " S, a $356 \mathrm{~m}$ de altitude), região norte do Estado de Mato Grosso. O clima da região é do tipo Tropical Quente e Úmido (Aw, segundo classificação de Köppen), com temperatura média anual de $24^{\circ} \mathrm{C}$, com pequena variação nas estações e uma precipitação média anual de 2000 mm (PRIANTE FILHO et al., 2004), sendo duas estações bem definidas, a estação chuvosa que corresponde ao período de outubro a abril e equivale a $80 \%$ do total de precipitação e a estação seca, de maio a setembro (GARRIDO, 2016). O plantio da cultivar Nanicão, possui área total de 2,00 hectares e 9 anos de implantação. As plantas encontram-se dispostas em fileiras duplas, com espaçamento de $1,75 \mathrm{~m}$ (na linha) por $2,50 \mathrm{~m}$ (entre linhas) por 4,50 m (entre as fileiras duplas), irrigadas por micro aspersão com turno de rega pré-definido.

$\mathrm{O}$ estudo foi desenvolvido no período de 12 meses, de maio de 2012 a abril de 2013, possibilitando desta maneira a observação do comportamento dos insetos no período seco e chuvoso da região. Durante o desenvolvimento do experimento, o bananal foi mantido sob os tratos culturais usualmente executados pelo produtor, como controle de matocompetição por roçagem entre as fileiras, desfolha, limpeza e desbaste de touceiras, adubação e irrigação. Todavia, não recebeu tratamento fitossanitário, minimizando assim a interferência externa nos dados.

Foram definidas duas parcelas experimentais, constituída de meio hectare cada, onde foram dispostas armadilhas tipo pitfall modificada. Estas foram confeccionadas a partir de garrafas plásticas transparentes do tipo Pet de dois litros, com duas aberturas laterais, compostas de dois cortes transversais de $10 \times 8 \mathrm{~cm}$, distanciados em $6,0 \mathrm{~cm}$ um do outro, formando uma "lingueta" dobrada para o lado de fora da garrafa. As mesmas foram mantidas com tampa, evitando entrada de água de chuva e/ou da irrigação.

$\mathrm{Na}$ parcela 1 foram instaladas duas armadilhas, utilizando como isca atrativa o feromônio sintético de agregação Cosmolure ${ }^{\circledR}$. As armadilhas foram dispostas aleatoriamente a $30 \mathrm{~m}$ de distância entre si, seguindo orientação do fabricante, e $30 \mathrm{~m}$ da borda do bananal. Estas foram instaladas no solo, com cerca de um terço da garrafa enterrada próxima ao pseudocaule das plantas, preservando as aberturas laterais das armadilhas a fim de exalar o feromônio e atrair os insetos.

O sachê de feromônio com concentração de $2,26 \mathrm{~g}_{\mathrm{kg}} \mathrm{kg}^{-1} \mathrm{de}$ (1S, 3R, 5R, 7S), 2,8-dioxa 1-etil 3,5,7-trimetil biciclo [3.2.1] octano, comumente conhecido como sordidina, substituído a cada 30 dias, foi acoplado nas armadilhas por meio de um arame suspenso com anzol na ponta, preso na tampa de cada garrafa. Foram adicionados ainda $150 \mathrm{~mL}$ de solução de água com detergente neutro na concentração de $5 \%$, com o intuito de reter os insetos atraídos para a armadilha, de acordo com a metodologia modificada de Reddy et al. (2009).

Na parcela 2, tratamento que utilizou como isca atrativa toletes de cana-de-açúcar, foram utilizadas 10 armadilhas com 3 toletes de $7 \mathrm{~cm}$ de cana-de-açúcar, devidamente amassados para facilitar a liberação dos voláteis. Os toletes de cana-deaçúcar eram substituídos a cada 15 dias, conforme metodologia utilizada por Soliman et al. (2009) e descartados longe da área experimental.

O intervalo de vistoria e coleta foi quinzenal, ocasião em que todo material presente nas armadilhas era cuidadosamente coletado com o auxílio de peneira plástica e pinça entomológica, acondicionado em potes plásticos, com álcool hidratado a 70\%, identificado com etiquetas e, em seguida encaminhado ao Laboratório de Microscopia para triagem, identificação por meio de chaves dicotômicas e contagem dos indivíduos. As armadilhas eram devidamente higienizadas e restauradas à condição ideal para nova coleta.

Após a identificação e contagem de todos os insetos coletados, em nível de ordem, separou-se os coleópteros $C$. sordidus e $M$. hemipterus, os quais foram identificados em nível de espécie, e convertidos em número de indivíduos por armadilha por mês.

A confirmação da identificação das espécies foi realizada pelo taxonomista Prof. Dr. Sérgio Antonio Vanin, da Universidade de São Paulo, e os exemplares ficaram depositados na coleção do Museu de Zoologia da USP. Vouchers dos mesmos também foram incorporados à coleção entomológica da Universidade Federal de Mato Grosso Campus de Sinop sob o número de depósito 506.

Dados meteorológicos, como precipitação pluviométrica, temperatura mínima, máxima, média e umidade relativa foram 
disponibilizados pela Estação Meteorológica da UFMT/Sinop (11 ${ }^{\circ} 51^{\prime} 49.28^{\prime \prime} \mathrm{S}, 5^{\circ} 29^{\prime} 8.07^{\prime \prime} \mathrm{O}$, a $\left.382 \mathrm{~m}\right)$, localizada no Campus da Universidade Federal de Mato Grosso, distante $7,70 \mathrm{~km}$ da área do experimento.

Os dados coletados no campo foram agrupados mensalmente, a frequência de captura foi analisada estatisticamente pelo Teste do Qui-quadrado e as variáveis climáticas pelo Teste F. A dinâmica populacional de $C$. sordidus e de $M$. hemipterus foi avaliada através de análise gráfica e de estudos de correlação linear simples entre o número do inseto por mês e os fatores climáticos (precipitação pluviométrica; temperaturas mínima, média e máxima; umidade relativa do ar).

\section{RESULTADOS}

O número médio mensal de $C$. sordidus coletado durante o período do estudo oscilou entre 5,5 indivíduos (mês de setembro 2012) a 48,5 (fevereiro de 2013) e em relação a $M$. hemipterus as médias mensais variaram entre 0,5 (maio/2012) e 13,9 adultos/isca por mês, no mês de pico populacional (novembro de 2012) (Tabela 1.).

Tabela 1. Número total e médias mensais de adultos de $C$. sordidus (COLEOPTERA: CURCULIONIDAE) capturados em armadilhas com isca de feromônio e $M$. hemipterus (COLEOPTERA: CURCULIONIDAE) capturados em armadilhas de toletes de canade-açúcar, no período de maio de 2012 a abril de 2013, em bananal da cv. Nanicão, em Sinop-MT.

Table 1. Total number and monthly averages of adults of $C$. sordidus caught in traps with pheromone bait and $M$. hemipterus caught in traps of sugarcane tops, from May 2012 to April 2013, in banana of cv. Nanicão in Sinop-MT.

\begin{tabular}{lcccc}
\hline \multicolumn{1}{c}{ Meses } & \multicolumn{2}{c}{ C. sordidus } & \multicolumn{2}{c}{ M. hemipterus } \\
\cline { 2 - 5 } 2012 a & Total & Média \pm DP & Total & Média \pm \\
\hline Maio & 12 & $6,00 \pm 8,49$ & 5 & $0,50 \pm 1,26$ \\
Junho & 15 & $3,75 \pm 3,10$ & 63 & $3,15 \pm 2,90$ \\
Julho & 16 & $4,00 \pm 2,83$ & 97 & $4,85 \pm 4,28$ \\
Agosto & 32 & $8,00 \pm 9,56$ & 72 & $3,60 \pm 3,70$ \\
Setembro & 11 & $2,75 \pm 4,86$ & 99 & $4,95 \pm 6,21$ \\
Outubro & 14 & $3,50 \pm 2,38$ & 33 & $1,65 \pm 1,69$ \\
Novembro & 83 & $13,83 \pm 18,55$ & 139 & $4,63 \pm 3,55$ \\
Dezembro & 17 & $4,25 \pm 3,10$ & 39 & $1,95 \pm 2,11$ \\
Janeiro & 58 & $14,50 \pm 8,54$ & 53 & $2,65 \pm 3,08$ \\
Fevereiro & 97 & $24,25 \pm 20,50$ & 10 & $0,50 \pm 0,94$ \\
Março & 53 & $13,25 \pm 15,44$ & 28 & $1,40 \pm 1,60$ \\
Abril & 34 & $8,50 \pm 7,94$ & 13 & $0,65 \pm 0,87$ \\
\hline Total & 442 & & 651 & \\
\hline
\end{tabular}

Média $=$ Total de adultos coletados por mês dividido pelo número de armadilhas com iscas de feromônio e de toletes de cana-de-açúcar; DP= Desvio padrão

Analisando a frequência de captura de $C$. sordidus durante todo o período do experimento observou-se que ocorreu variação na densidade populacional do inseto nas duas épocas do ano, segundo Teste do Qui-quadrado $(\mathrm{p}<0,05)$, situação contrária ao verificado quanto a $M$. hemipterus, onde o Teste do Qui-quadrado $(\mathrm{p}>0,05)$ indicou que não houve diferença significativa na densidade populacional deste inseto nas diferentes épocas do ano (Tabela 2).

A dinâmica populacional de $C$. sordidus apresentou picos populacionais nos meses de maior precipitação pluviométrica na região - novembro de $2012(275,90 \mathrm{~mm})$, janeiro $(210,00$ $\mathrm{mm}$ ) e fevereiro de 2013 (404,60 mm), com médias de 41,50, 29 e 48,50 adultos por isca por mês, respectivamente. Os meses de menor média de ocorrência do inseto foram aqueles de baixa precipitação - maio, junho e setembro de 2012 com 6, 7,50 e 5,50 adultos por isca por mês e 54,20, 37,60 e 5,20 mm de precipitação pluviométrica, respectivamente (Figura 1).

Tabela 2. Total de adultos de $C$. sordidus e $M$. hemipterus capturados e suas respectivas frequências em relação às épocas do ano, no período de maio de 2012 a abril de 2013, em bananal da cv. Nanicão, em Sinop-MT.

Table 2. Total adults of C. sordidus and M. hemipterus captured and their respective frequencies in relation to the seasons of the year, from May 2012 to April 2013, in banana from cv. Nanicão in Sinop-MT.

\begin{tabular}{lcc}
\hline Época & C. sordidus* & M. hemipterus** \\
\hline Chuvosa & $356(80,54 \%)$ & $315(48,39 \%)$ \\
Seca & $86(19,46 \%)$ & $336(56,61 \%)$ \\
Total & $442(100 \%)$ & $651(100 \%)$ \\
\hline
\end{tabular}

*Teste do Qui-quadrado a 5\% ( $\left.\chi^{2}\right)=164,93 ; p=0,0001$

** Teste do Qui-quadrado a $5 \%\left(\chi^{2}\right)=0,67 ; p=0,41$

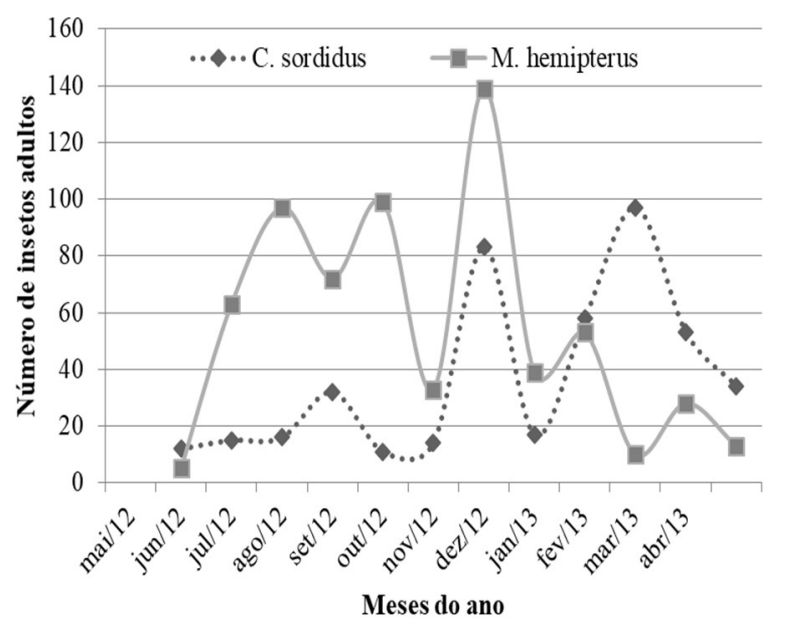

Figura 1. Flutuação populacional de adultos de C. sordidus capturados em armadilhas com isca de feromônio e $M$. hemipterus capturados em armadilhas de toletes de cana-de-açúcar, no período de maio de 2012 a abril de 2013, em bananal da cv. Nanicão, em Sinop-MT- Brasil.

Figure 1. Population fluctuation of adults of $C$. sordidus caught in traps with pheromone bait and $M$. hemipterus caught in traps of sugarcane tops, from May 2012 to April 2013, in banana from cv. Nanicão, in Sinop-MT- Brazil.

No estudo de correlação dos fatores ambientais (temperaturas média, máxima e mínima; precipitação pluviométrica e umidade relativa do ar) constatou-se uma forte correlação linear simples positiva entre o número de $C$. sordidus capturados e a precipitação pluviométrica (Tabela 3.). Portanto, à medida que aumentou a precipitação pluviométrica ocorreu aumento na população do inseto no bananal (Figura 2).

As correlações entre o número de $M$. hemipterus e as variáveis climáticas foram todas não significativas, consequentemente a flutuação populacional deste inseto ocorreu de maneira estatisticamente independente das variáveis climáticas.

\section{DISCUSSÃO}

Em todas as avaliações a população de adultos de C. sordidus apresentou altos índices mensais, superando na maioria dos meses o nível de controle, que segundo Gallo et 
al. (2002) é de 5 adultos por isca por mês. Estes resultados refletiram o alto grau de infestação de C. sordidus no local com consequente danos e prejuízos a produção, apesar destas duas variáveis não terem sido avaliadas. Dantas et al. (2011) relacionaram a cultivar Nanicão dentre as mais susceptíveis ao moleque-da-bananeira, o que pode justificar o alto índice de infestação no cultivo avaliado. Entretanto, um importante fator a se analisar é o de que na área do experimento nenhum monitoramento ou controle havia sido realizado nos últimos dois anos, devido ser considerado pelo proprietário como um bananal com plantas em final de produção e, portanto, em vias de ser reformado. Outra condição que pode ter contribuído para esta alta infestação seria o excesso de cobertura morta encontrado na área, que pode ter servido de abrigo à proliferação de C. sordidus, conforme relataram Borges et al. (2015).

Tabela 3. Equações de regressão e coeficientes de correlação encontrados entre os fatores climáticos e o número mensal de adultos de C. sordidus, de maio de 2012 a abril de 2013, em bananal da cv. Nanicão, em Sinop-MT

Table 3. Regression equations and correlation coefficients found between climatic factors and the monthly number of adults of $C$. sordidus, from May 2012 to April 2013, in banana from cv. Nanicão in Sinop-MT

\begin{tabular}{lcc}
\hline $\begin{array}{c}\text { Fatores } \\
\text { climáticos }\end{array}$ & Equações de regressão & $\begin{array}{c}\text { Coef. de } \\
\text { Correlação "r" }\end{array}$ \\
\hline T média $\left({ }^{\circ} \mathrm{C}\right)$ & $\mathrm{y}=89,8940-14,1946 \mathrm{x}$ & $0,44364 \mathrm{~ns}$ \\
T máx $\left({ }^{\circ} \mathrm{C}\right)$ & $\mathrm{y}=-2862,6416+166,2898$ & $-0,28189 \mathrm{~ns}$ \\
T mín $\left({ }^{\circ} \mathrm{C}\right)$ & $\mathrm{y}=423,3149+54,7709 \mathrm{x}$ & $0,56438 \mathrm{~ns}$ \\
P pluvial $(\mathrm{mm})$ & $\mathrm{y}=17,1781-0,0018 \mathrm{x}$ & $0,77393 *$ \\
UR & $\mathrm{y}=408,1960-12,4138 \mathrm{x}$ & $0,51152 \mathrm{~ns}$ \\
\hline
\end{tabular}

*correlação significativa ao nível de $5 \%$ de probabilidade; ns= correlação não significativa

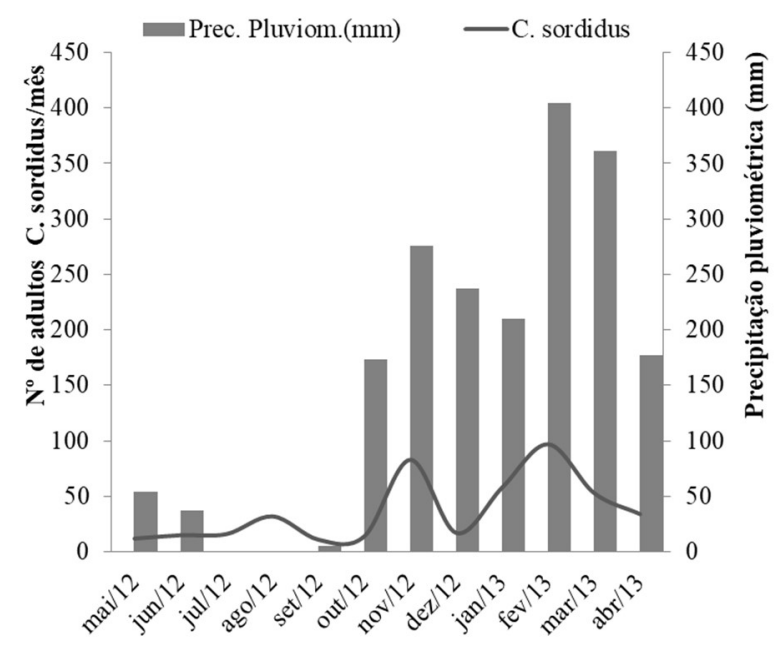

Figura 2. Adultos de C. sordidus capturados em armadilhas com isca de feromônio e precipitação pluviométrica, no período de maio de 2012 a abril de 2013, em bananal da cv./ Nanicão, em Sinop-MTBrasil.

Figure 2. Adults of $C$. sordidus captured in traps with pheromone bait and rainfall, from May 2012 to April 2013, in bananal of cv./ Nanicão, in Sinop-MT- Brazil.

M. hemipterus é frequentemente citado por não causar dano econômico em bananais, pois se alimenta comumente de pseudocaules tombados, em decomposição ou de plantas debilitadas, entretanto Fancelli et al. (2012), em experimento com a bananeira cultivar Terra, registraram um índice médio de captura de 0,54 insetos/isca/coleta semanal, em armadilhas de pseudocaule, provocando sérios danos ao cultivo, por broquearem as plantas a uma altura entre aproximadamente 0,2 $\mathrm{m}$ e 1,0 $\mathrm{m}$ do nível do solo, levando as plantas ao tombamento, número bastante inferior ao pico populacional de 13,9 adultos/isca/mês registrado neste estudo, portanto a situação encontrada alerta para uma observação mais ponderada deste inseto. Contudo, Pauli et al. (2011) ressaltam o importante papel da espécie como disseminador do fungo Beauveria bassiana, contribuindo com o controle biológico para $C$. sordidus.

A maior incidência de adultos de C. sordidus foi registrada nos meses de maior precipitação (novembro a fevereiro), diferindo do resultado encontrado por Malagodi-Braga et al. (2014), que avaliaram a flutuação populacional de $C$. sordidus em um bananal em transição agroecológica, das cultivares 'Prata Conquista', 'Prata maravilha' e 'Prata' na cidade de Jaguariúna- SP, no qual a correlação entre precipitação pluviométrica e número de indivíduos adultos nas armadilhas foi negativa, com pico populacional nos meses de agosto e setembro, que correspondem ao período de estiagem no local. Entretanto, os autores ressaltaram ainda que, o número de insetos coletados ficou abaixo do nível de dano econômico. Pavarini et al. (2009), em estudos com isca de pseudocaule da cultivar Nanica, no município de Juquiá-SP, também verificaram a maior incidência de $C$. sordidus em meses de baixa precipitação pluviométrica e temperaturas amenas. Os autores justificaram esta situação devido ao inseto ter afinidade pela umidade e nos períodos de baixas precipitações as iscas tornarem-se os únicos locais úmidos no bananal, portanto atrativos ao inseto.

Segundo Gallo et al. (2002) a distribuição da população, assim como a quantidade de insetos de $C$. sordidus são diretamente dependentes de fatores ambientais, entre eles a temperatura e umidade relativa do ar.

Possivelmente as variações observadas nos picos populacionais de C. sordidus obtidos neste estudo e dos demais pesquisadores foram decorrentes não apenas de fatores bióticos, como também de fatores abióticos específicos de cada local de realização dos estudos.

\section{CONCLUSÕES}

Foi constatado o alto índice populacional de C. sordidus no cultivo durante o período avaliado, portanto a população atingiu o status de praga. Sua dinâmica populacional, foi influenciada positivamente pela precipitação pluviométrica, apresentando maiores níveis populacionais na época chuvosa, com picos nos meses de novembro de 2012, janeiro e fevereiro de 2013. Conhecer a época de maior incidência da praga permite um melhor planejamento de seu controle, o qual deverá deve ser realizado mais intensamente neste período, minimizando danos ao cultivo, prejuízos econômicos e riscos ao ambiente.

Quanto a M. hemipterus observou-se que a população do inseto não sofreu qualquer influência de variáveis climáticas, com distribuição equilibrada ao longo do ano.

\section{AGRADECIMENTOS}

Ao taxonomista Prof. Dr. Sérgio Antônio Vanin, do Museu de Zoologia do Instituto de Biociências, da Universidade de São Paulo (USP) pela confirmação das espécies estudadas e, a Família Bianchi por disponibilizar a área para a instalação e desenvolvimento do experimento. 


\section{REFERÊNCIAS}

BORGES, A. L.; MATOS, A. P.; RITZINGER, C. H. S. P.; SOUZA, L. S.; LIMA, M. B.; FANCELLI, M.; CORDEIRO, Z. J. M. Boas Práticas Agrícolas de Campo no Cultivo de Bananeira. Cruz das Almas: Embrapa Mandioca e Fruticultura, 2015. 52 p. (Documentos, 214)

DANTAS, D. J.; MEDEIROS, A. C.; NUNES, G. H. S.; MENDONÇA, V.; MOREIRA, M. A. B. Reação de cultivares de bananeira ao Cosmopolites sordidus no Vale do Açu - RN. Revista Verde de Agroecologia e Desenvolvimento Sustentável, Mossoró, v. 6, n. 3, p.152155, jul./set. 2011.

DIONISIO, L. F. S.; LIMA, A. C. S.; MORAIS, E. G. F.; CORREIA, R. G.; SANTOS, A. V. F.; XIMENES, C. K. S. Distribuição espacial de Metamasius hemipterus (Coleoptera: Curculionidae) em plantio de Dendê (Elaeis guianeensis Jacq) em Roraima. Revista Agroambiente On-line, Boa Vista, v. 9, n. 3, p.327-336, jul./set. 2015. DOI: 8470ragro.v9i3.2517 http://dx.doi.org/10.18227/1982-

FANCELLI, M.; BORGES, A. L.; RITZINGER, C. H. S. P.; SILVA, D. S.; RINGENBERG, R. Metamasius hemipterus L. como praga de bananeiras cv. Terra. Revista Brasileira de Fruticultura, Jaboticabal, v. 34, n. 3, p. 944-946, set. 2012. DOI: http://dx.doi.org/10.1590/S010029452012000300039

FANCELLI, M.; MILANEZ, J. M.; MESQUITA, A. L. M.; COSTA, A. C. F.; COSTA, J. N. M.; PAVARINI, R.; PAVARINI, G. M. P. Artrópodes: pragas da bananeira e controle. Informe Agropecuário, Belo Horizonte, v. 36, n. 288 , p. 7-18, 2015.

FAO_FOOD AND AGRICULTURE ORGANIZATION OF THE UNITE NATIONS. FAO Statistical Databases. 2015 . Disponível em: $<$ https:/www.iaea.org/about/partnerships/food-andagriculture-organization?>. Acesso em: 17/08/2015.

GALLO, D. NAKANO, O.; SILVEIRA NETO, S.; CARVALHO, R. P. L.; BATISTA, G. C. de; BERTI FILHO, E.; PARRA, J. R.P.; ZUCCHI, R. A.; ALVES, S. B.; VENDRAMIN, J. D.; MARCHINI, L. C.; LOPES, J. R. S.; OMOTO, C. Manual de Entomologia agrícola. 3.ed. São Paulo: Agronômico Ceres, 2002. 920 p.

GARRIDO, G. Estudo do aporte de nutrientes via serapilheira em um ecótono de floresta tropical e cerrado na região de Sinop- MT. 2016. 52f. Dissertação (Mestrado em Ciências Ambientais) - Universidade de Cuiabá, Cuiabá, 2016.

IBGE INSTITUTO BRASILEIRO DE GEOGRAFIA E ESTATÍSTICA (IBGE). Levantamento Sistemático da Produção Agrícola: Pesquisa Mensal de Previsão e Acompanhamento das Safras Agrícolas no Ano Civil. Rio de Janeiro, v. 30, n. 1, p.1-81, janeiro.2017.

KOCHEMBORGER, J.; LOPES, L. M.; SILVA JÚNIOR, E. C.; SOUSA, A. H. Ocorrência de Metamasius hemipterus (Coleoptera: Curculionidae) em cultivos de banana no município de Senador Guiomard, Acre, Brasil. Revista de Biologia e Ciências da Terra, Aracaju, v. 16, n. 2, p. 1416,2016
MALAGODI-BRAGA, K. S.; MORICONI, W.; SILVA, R. F.; NEVES, M. C.; CORRALES, F. M.; QUEIROGA, J. L. Incidência do Moleque-da-bananeira em uma Área Degradada em Transição Agroecológica. Cadernos de Agroecologia, Dourados, v. 19, n. 4, p. 1-8, nov. 2014.

MOLIN, I. L. D.; BARRETO, M. R. Ocorrência e controle de Curculionidae em Cocos nucifera L. em Sinop - Mato Grosso. Semina: Ciências Biológicas e da Saúde, Londrina, v. 33, n. 1, p. 53-64, jan./jun. 2012. DOI: http://dx.doi.org/10.5433/1679-0367.2012v33n1p53

PAULI, G.; LOPES, R. B.; ALVES, S. B.; DAMATTO JUNIOR, E. R.; MASCARIN, G. M. Falsa broca aumenta disseminação de Beauveria bassiana em populações de campo da broca-do-rizoma da bananeira. Ciência Rural, Santa Maria, v. 41, n. 11, p. 1867-1870, nov. 2011. DOI:

PAVARINI, R.; PAVARINI, G. M. P.; GOMES, J. M.; PINOTTI, E. B.; MARZOLA, D.; RESENDE, R. Influência das diferentes estações do ano na ocorrência de adultos de Cosmopolites sordidus (Coleoptera: Curculionidae). Revista Científica Eletrônica de Agronomia, Garça, v. 16, p. 07-11, dez. 2009.

PRIANTE FILHO, N.; VOURLITIS, G. L.; HAYASHI, M. M. S.; NOGUEIRA, J. S.; CAMPELO JR, J. H.; NUNES, P. C.; SANCHES E SOUZA, L.; COUTO, E. G.; HOEGER, W.; RAITER, F.; TRIENWEILER, J. L.; MIRANDA, E. J.; PRIANTE, P. C.; FRITZEN, C. L.; LACERDA, M.; PEREIRA, L. C.; BIUDES, M. S.; SULI, G. S.; SHIRAIWA, S.; PAULO, S. R.; SILVEIRA, M. Comparason of the mass and energy exchange of a pasture and a mature transitional tropical forest of the southern Amazon basin during the wet-dry season transition. Global Change Biology, Oxford, v. 10, n. 5, p. 863-876, 2004. DOI: https://dx.doi.org/10.1111/j.15298817.2003.00775.x

REDDY, G. V. P.; CRUZ, Z. T.; GUERRERO, A. Development of an efficient pheromone-based trapping method for the banana root borer Cosmopolites sordidus. Journal of Chemical Ecology, New York, v. 35, n. 1, p. 111-117, 2009. DOI: http://dx.doi.org/10.1007/s10886008-9580-6

SOLIMAN, E. P.; PAVARINI, R.; GARCIA, V. A.; LIMA, R. C.; NOMURA, E. S.; PAVARINI, G. M. P.; DAMATTO, E. R. Diferentes iscas atrativas para o monitoramento populacional de Metamasius sp. (Coleoptera: Curculionidae) no cultivo da pupunheira. Revista Eletrônica de Agronomia, Garça, v. 16, n. 2, p. 1-6, dez. 2009.

ZORZENON, F. J.; BERGMAN, E. C.; BICUDO, J. E. A. Primeira ocorrência de Metamasius hemipterus (Linnaeus, 1758) e Metamasius ensirostris (germar, 1824) (Coleoptera, Curculionidae) em palmiteiros dos gêneros Euterpe e Bactris (Arecaceae) no Brasil. Arquivos do Instituto Biológico, São Paulo, v. 67, n. 2, p. 265-268, jul./dez. 2000. 